\title{
Superior Mesenteric Vein Thrombosis as an Early Presentation of Myelodysplastic Syndrome: A Case Report
}

\author{
Min Jung Kim, Hyun Joo Song ${ }^{1}$, Woo Kun $\mathrm{Kim}^{1}$, Sun Hyung Kim ${ }^{2}$ \\ Departments of Surgery, Internal Medicinel, and Laboratory Medicine², Jeju National University School of Medicine, Jeju, Korea
}

\begin{abstract}
Mesenteric venous thrombosis (MVT) is a serious condition due to its potential association with mesenteric ischemia and infarction of the small bowel. Symptoms of MVT are often vague, making accurate diagnosis and sufficient treatment difficult. However, increased awareness and new imaging modalities for this condition have improved outcomes for patients with MVT. Treatment includes anticoagulation, transcatheter therapy, and surgery. In the present report, we describe the case study of a 62-year-old woman with a presenting diagnosis of superior MVT, who was finally diagnosed with myelodysplastic syndrome. The superior MVT spontaneously dissolved after the patient underwent 6 months of systemic anticoagulation therapy. Invasive surgery or bowel resection was not required. (Intest Res 2014;12:157-161)
\end{abstract}

Key Words: Mesenteric vein; Thrombosis; Myelodysplastic syndrome

\section{INTRODUCTION}

In cases of acute mesenteric ischemia, $5-15 \%$ cases are caused by superior mesenteric vein (SMV) thrombosis. ${ }^{1}$ In cases of mesenteric vein thrombosis (MVT), intestinal ischemia can occur if there is mesenteric venous blood flow obstruction, resulting in critical bowel wall edema and fluid efflux into the lumen. This can result in diminished arterial blood flow, leading to submucosal hemorrhage and bowel infarction. MVT can be fatal if the diagnosis and subsequent treatment is delayed. ${ }^{2}$

In the present report, we describe the case of a patient with SMV thrombosis who presented with abdominal pain and was treated with systematic anticoagulation therapy for

Received September 25, 2013. Revised November 2, 2013.

Accepted November 4, 2013.

Correspondence to Hyun Joo Song, Department of Internal Medicine, Jeju National University Hospital, 15 Aran 13-gil, Jeju 690-767, Korea. Tel: +8264-754-8142, Fax: +82-64-717-1131, E-mail: songhj@jejunu.ac.kr

Financial support: This research was supported by the 2014 scientific promotion program funded by Jeju National University.

Conflict of interest: None. a 6-month period. Myelodysplastic syndrome (MDS) was diagnosed during the work-up that was performed to identify the cause of the SMV thrombosis.

\section{CASE REPORT}

A 62-year-old woman who was admitted to the hospital presented with a 7-day history of abdominal pain, which had worsened over time and was associated with postprandial bloating. She had experienced sudden weight gain of approximately $3 \mathrm{~kg}$ in the past month. The patient had been diagnosed with rheumatoid arthritis 7 years previously and was taking the following medications: low-dose glucocorticoids such as prednisolone ( $5 \mathrm{mg} /$ day) and methotrexate (15 mg/week), as well as the nonsteroidal anti-inflammatory drug celecoxib (200 mg/day). The patient had no history of surgery or trauma, and there was no family history of venous thromboembolism. On admission, the patient had an elevated temperature of $38.5^{\circ} \mathrm{C}$ and abdominal tenderness without muscle guarding.

Initial laboratory findings revealed pancytopenia (hemo-

๑ Copyright 2014. Korean Association for the Study of Intestinal Diseases. All rights reserved.

This is an Open Access article distributed under the terms of the Creative Commons Attribution Non-Commercial License (http://creativecommons.org/licenses/by-nc/3.0)

which permits unrestricted non-commercial use, distribution, and reproduction in any medium, provided the original work is properly cited. 
globin concentration, $9.8 \mathrm{~g} / \mathrm{dL}$; white blood cell count, 3,100/ $\mathrm{mm}^{3}$; and platelet count, $32,000 / \mathrm{mm}^{3}$ ). The prothrombin time (international normalized ratio, 1.43) and activated partial thromboplastin time (42 seconds) were prolonged.
The levels of fibrinogen and fibrinogen degradation product were $186 \mathrm{mg} / \mathrm{dL}$ and $>20 \mu \mathrm{g} / \mathrm{mL}$, respectively, whereas the D-dimer level was elevated by $6 \mu \mathrm{g} / \mathrm{mL}$. Moreover, evidence of disseminated intravascular coagulation was observed.
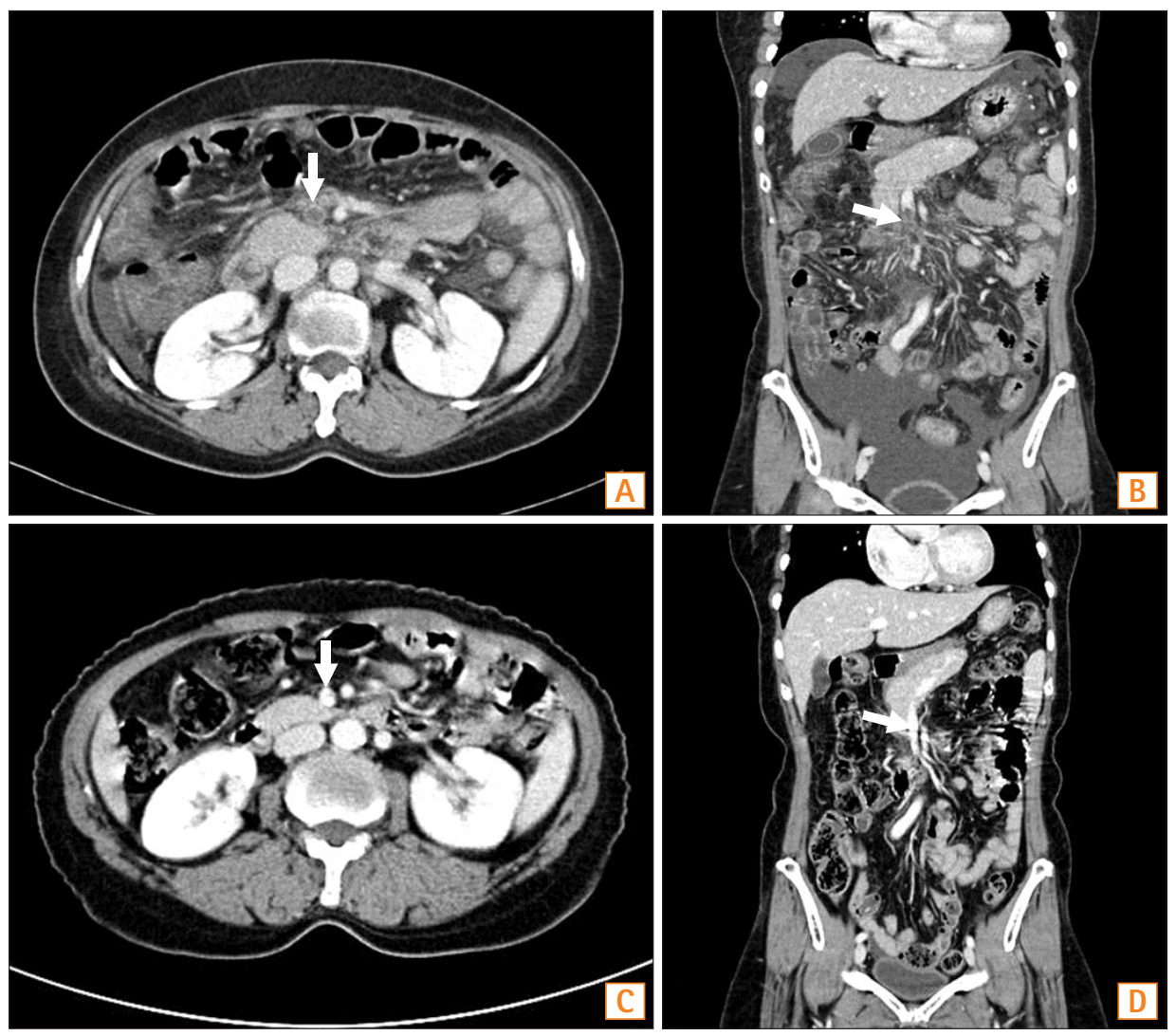

Fig. 1. Abdominal-pelvic CT findings. (A) At admission, axial CT showed thrombosis (white arrow) in the superior mesenteric vein (SMV) and its tributary. (B) Coronal CT showed a moderate amount of ascites with omental and mesenteric infiltrations associated with SMV thrombosis; the thrombus was $4.5 \mathrm{~cm}$ in length (white arrow). (C) CT findings after 6 months of anticoagulation therapy showed complete resolution of the SMV thrombosis (white arrow), (D) coronal CT image after 6 months of anticoagulation therapy.
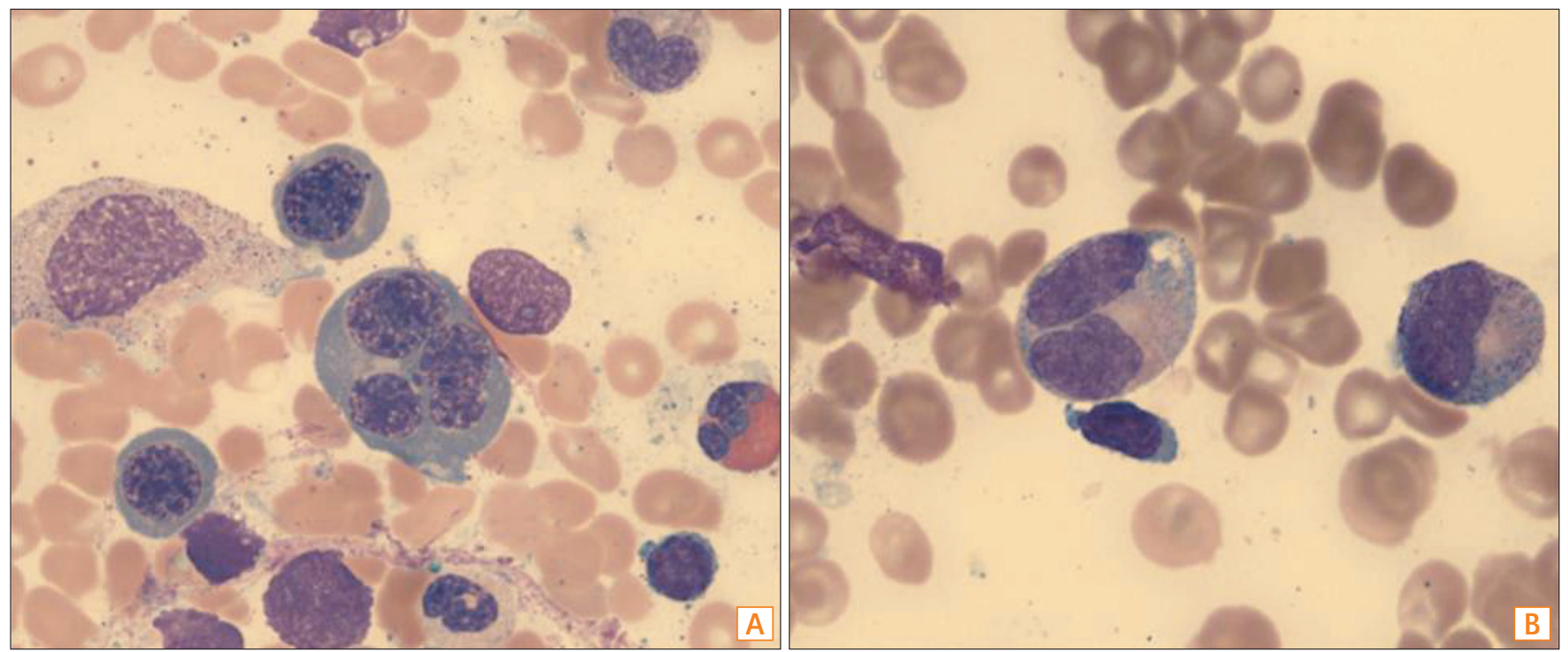

Fig. 2. Bone marrow aspirate smear (Wright stain, $\times 1000)$. (A) Dysplastic erythroid precursors with multinuclearity and megaloblastic changes. (B) Dysplastic large neutrophils with bilobed nuclei. 

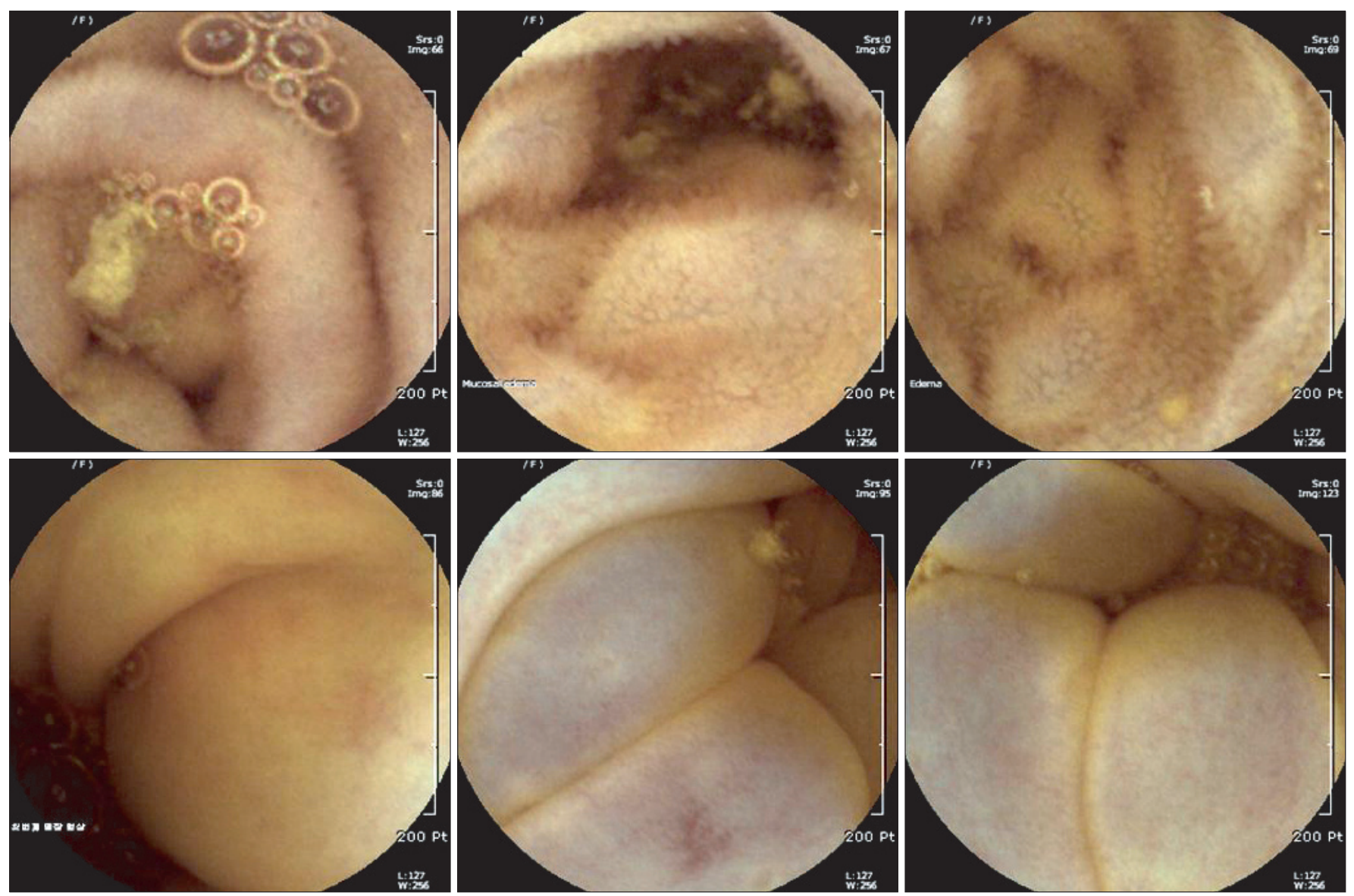

Fig. 3. Capsule endoscopic (CE) findings. CE findings showed moderate small bowel wall edema from the distal jejunum to the ileum. Severe mucosal edema with erythematous patches in the ascending colon was also observed.

CT scan of the abdomen and pelvis showed a moderate amount of ascites with omental and mesenteric infiltration, which was associated with a $4.5-\mathrm{cm}$ thrombus in the SMV and its tributary (Fig. 1A, B). Additional laboratory work-up was also performed. The serum lactate concentration was $<1.0 \mathrm{mmol} / \mathrm{L}$. No antinuclear or anticardiolipin antibodies were detected. In addition, there was no evidence of paroxysmal nocturnal hemoglobinuria, antiphospholipid syndrome, or other autoimmune disease. The antithrombin III level was $50 \%$. The protein C concentration (46\%) and S activity (26\%) were below the normal ranges.

Anticoagulation therapy was initiated, and the patient was closely monitored for signs of bowel infarction. Intravenous hydration and broad-spectrum prophylactic antibiotics were administered. Moreover, low-molecular-weight heparin was initiated. On the third day of admission, the patient's bone marrow was examined in order to identify the cause of the pancytopenia and the recent thrombotic event. A diagnosis of MDS was made, which was believed to involve refractory cytopenia with multilineage dysplasia (Fig. 2).
Thereafter, the patient experienced sustained hematochezia and vague abdominal pain. On the fourth day, capsule endoscopy was performed to exclude bowel ischemia. Through small bowel endoscopy, we identified the presence of diffuse mucosal edema from the distal jejunum to terminal ileum and erythematous patches in the ascending colon, which were compatible with SMV territories (Fig. 3). There was no evidence of ischemic enteritis. The patient demonstrated improvement after oral anticoagulants were administered during her 12-day hospital admission. After 6 months of anticoagulation therapy, follow-up CT of the abdomen and pelvis showed complete dissolution of the SMV thrombosis (Fig. 1C, D). The laboratory findings also improved. The patient experienced no recurrence of SMV thrombosis during the 2-year follow-up period.

\section{DISCUSSION}

Acute MVT is a rare but potentially fatal condition, with a mortality rate of $20-30 \%{ }^{3,4}$ However, diagnosis of this con- 
dition is difficult because the symptoms are nonspecific. The most commonly reported symptom is abdominal pain. Other symptoms include abdominal distension, nausea, and fever. Abdominal symptoms may gradually worsen with the development of intestinal infarction. Critical symptoms such as hemodynamic instability and peritonitis are important indicators as to whether surgery is required. Suspicion of MVT is important for avoiding delays in diagnosis and therapy, particularly in elderly patients.

The development of imagining modalities over the past 2 decades has improved the ability to make an accurate diagnosis of MVT. In this patient, SMV thrombosis was diagnosed easily because of contrasted CT. Portal venous phase $\mathrm{CT}$ is the most sensitive technique for diagnosing MVT. The sensitivity of diagnosis by contrast-enhanced abdominal CT is approximately $90 \%{ }^{2}$

MVT is classified as either primary or secondary. Primary SMV thrombosis is idiopathic, whereas secondary SMV thrombosis can result from various underlying causes, including hypercoagulable states, pregnancy, or recent abdominal surgery. ${ }^{5}$ After an MVT diagnosis is made, the clinician should attempt to identify the cause of the thrombosis. Our patient had been diagnosed with rheumatoid arthritis 7 years prior to the current admission. Reports have shown that there is an increased risk of developing deep vein thrombosis in patients with rheumatoid arthritis compared with patients who did not have rheumatoid arthritis. ${ }^{6}$ However, whether treatment or disease activity can modify the risk of venous thromboembolism in patients with rheumatoid arthritis remains controversial. ${ }^{7}$ Slight decreases in antithrombin III and protein C levels, and S activity were identified in the present case. After a hematological specialist was consulted, these findings were confirmed to be the result-as opposed to the cause-of the recent massive thrombosis. The pancytopenia that was noted on peripheral blood examination and multilineage dysplasia indicated by the assessment of bone marrow aspirate led to the diagnosis of MDS. MDS is a clonal hematological disorder that involves ineffective hematopoiesis.

Although myeloproliferative disease and malignancy are the most frequently diagnosed thrombophilic conditions, ${ }^{8,9}$ other studies have reported the presence of thrombosis in association with MDS, as in the present case. ${ }^{10,11}$ Kimura et al. ${ }^{12}$ found that patients with MDS and trisomy 8 were more likely to develop thrombosis and intestinal ulcers. Chen et al. ${ }^{11}$ reported a case of large-vessel thrombosis that was complicated by MDS and trisomy 8 . Although no definite cause of thrombosis has been identified, thrombosis and hemor- rhage complicated by MDS most likely occur as a result of quantitative and qualitative platelet anomalies. ${ }^{10}$ Other researchers have noted that in cases of MDS, immunological disorders caused by neutrophil dysfunction and inflammatory cytokine overproduction may lead to injury and inflammation of the endothelium. ${ }^{11,13,14}$ There have been reports of patients with MDS and autoimmune disease, including Behçet's disease, particularly in Japan. ${ }^{15,16}$

Another interesting point of our case is the capsule endoscopic findings. To our knowledge, capsule endoscopic findings have not been reported for SMV thrombosis. ${ }^{17}$ The patient experienced sustained symptoms during initial anticoagulation therapy, and bowel ischemia should be excluded in such cases. Therefore, capsule endoscopy can play a major role as a noninvasive procedure in cases where clinicians are determining whether surgery is warranted, particularly in cases involving sustained symptoms or controversial imaging findings.

A patient's clinical status typically determines the initial management of MVT. If the patient is clinically unstable, immediate surgical exploration is warranted. If a patient is clinically stable, conservative management, including systemic anticoagulation, is considered appropriate. ${ }^{18,19}$ Catheterdirected thrombolysis is a safe and effective treatment in cases of main trunk involvement. ${ }^{20}$ The typical duration of anticoagulation treatment is $6-12$ months. ${ }^{3}$ The immediate initiation of anticoagulation with low-molecular-weight heparin and a subsequent 6-month course of warfarin proved to be successful in the present case.

This case demonstrates the importance of early detection of SMV thrombosis followed by active systemic anticoagulation. Thus, we believe that clinicians should adopt a high degree of suspicion in such cases, and this increased awareness along with the early initiation of systemic anticoagulation therapy are essential for successful treatment of MVT.

\section{REFERENCES}

1. Kumar S, Sarr MG, Kamath PS. Mesenteric venous thrombosis. N Engl J Med 2001;345:1683-1688.

2. Singal AK, Kamath PS, Tefferi A. Mesenteric venous thrombosis. Mayo Clin Proc 2013;88:285-294.

3. Kaminsky M, Hochman D. Superior mesenteric venous thrombosis. CMAJ 2011;183:693.

4. Abu-Daff NS, Abu-Daff SN, Rubayaan A, Abu-Shaaban A. Laparoscopy in the treatment of a giant true epiphrenic diverticulum with migration of the gastrointestinal anastomosis staples. Saudi Med J 2009;30:295-298. 
5. Schoots IG, Levi M, van Vliet AK, Declerck PJ, Maas AM, van Gulik TM. Enhancement of endogenous fibrinolysis does not reduce local fibrin deposition, but modulates inflammation upon intestinal ischemia and reperfusion. Thromb Haemost 2004;91:497-505.

6. Kang JH, Keller JJ, Lin YK, Lin HC. A population-based casecontrol study on the association between rheumatoid arthritis and deep vein thrombosis. J Vasc Surg 2012;56:1642-1648.

7. Kim SC, Schneeweiss S, Liu J, Solomon DH. Risk of venous thromboembolism in patients with rheumatoid arthritis. Arthritis Care Res (Hoboken) 2013;65:1600-1607.

8. Cenedese A, Monneuse O, Gruner L, Tissot E, Mennesson N, Barth X. Initial management of extensive mesenteric venous thrombosis: retrospective study of nine cases. World J Surg 2009;33:2203-2208.

9. Zhang J, Duan ZQ, Song QB, Luo YW, Xin SJ, Zhang Q. Acute mesenteric venous thrombosis: a better outcome achieved through improved imaging techniques and a changed policy of clinical management. Eur J Vasc Endovasc Surg 2004;28:329334.

10. Landolfi R, Di Gennaro L. Thrombosis in myeloproliferative and myelodysplastic syndromes. Hematology 2012;17(Suppl 1):S174-S176.

11. Chen HC, Chiu YM. Large-vessel thrombosis in intestinal Behcet's disease complicated with myelodysplastic syndrome and trisomy 8. World J Gastroenterol 2012;18:1137-1140.

12. Kimura S, Kuroda J, Akaogi T, Hayashi H, Kobayashi Y, Kondo M. Trisomy 8 involved in myelodysplastic syndromes as a risk factor for intestinal ulcers and thrombosis--Behcet's syndrome.
Leuk Lymphoma 2001;42:115-121.

13. Hsu HC, Lee YM, Tsai WH, et al. Circulating levels of thrombopoietic and inflammatory cytokines in patients with acute myeloblastic leukemia and myelodysplastic syndrome. Oncology 2002;63:64-69.

14. Martin S, Baldock SC, Ghoneim AT, Child JA. Defective neutrophil function and microbicidal mechanisms in the myelodysplastic disorders. J Clin Pathol 1983;36:1120-1128.

15. Tada Y, Koarada S, Haruta Y, Mitamura M, Ohta A, Nagasawa K. The association of Behcet's disease with myelodysplastic syndrome in Japan: a review of the literature. Clin Exp Rheumatol 2006;24:S115-S119.

16. Kawabata H, Sawaki T, Kawanami T, et al. Myelodysplastic syndrome complicated with inflammatory intestinal ulcers: significance of trisomy 8. Intern Med 2006;45:1309-1314.

17. Kumar S, Kamath PS. Acute superior mesenteric venous thrombosis: one disease or two? Am J Gastroenterol 2003;98:12991304.

18. Brunaud L, Antunes L, Collinet-Adler S, et al. Acute mesenteric venous thrombosis: case for nonoperative management. J Vasc Surg 2001;34:673-679.

19. Acosta D, Rottbeck R, Rodriguez G, Ferri CP, Prince MJ. The epidemiology of dependency among urban-dwelling older people in the Dominican Republic; a cross-sectional survey. BMC Public Health 2008;8:285.

20. Wang MQ, Liu FY, Duan F, Wang ZJ, Song P, Fan QS. Acute symptomatic mesenteric venous thrombosis: treatment by catheter-directed thrombolysis with transjugular intrahepatic route. Abdom Imaging 2011;36:390-398. 